\title{
Cardiac Biphasic Synovial Sarcoma
}

National Cancer Institute

\section{Source}

National Cancer Institute. Cardiac Biphasic Synovial Sarcoma. NCI Thesaurus. Code C45757.

A biphasic synovial sarcoma arising from the heart. 